\title{
Analysis of CrossCheck Data on Two Years' Worth of Papers Submitted to Archives of Plastic Surgery
}

\author{
Jang Hyun Lee
}

Deputy Editor, Archives of Plastic Surgery

Department of Plastic and Reconstructive Surgery, Hanyang University Guri Hospital, Hanyang University College of Medicine, Guri, Korea

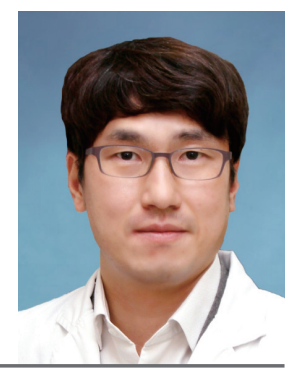

All manuscripts submitted to the Archives of Plastic Surgery are run through CrossCheck using the software powered by iThenticate to compare their similarity with previously published papers [1]. The editors use the results of this comparison for reference in manuscript screening. The results include the similarity index, which represents the overall similarity of a submitted manuscript to previously published papers.

The author analyzed the similarity index of the manuscripts submitted to the Archives of Plastic Surgery for two years from the year 2012 to 2013, a total of 440 manuscripts: 289 manuscripts in 2012 and 151 manuscripts in 2013. The overall

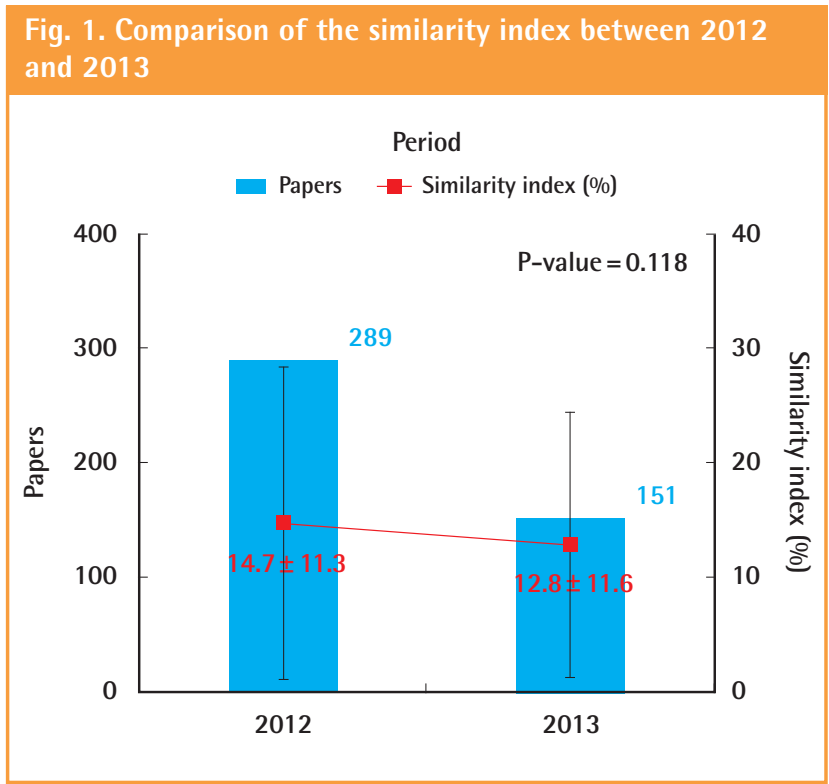

similarity index of 2013 fell from 2012 (Fig. 1). Although this decrease was not statistically significant, we assume the reason for the decrease was the introduction of the use of CrossCheck for authors. The similarity index of the manuscripts that were accepted was lower than that of the manuscripts that were not accepted ( $\mathrm{P}=0.046)$ (Fig. 2). This implies that manuscripts accepted for publication are likely to be of better quality. The similarity index of papers submitted from English-dominant countries was lower than that for authors from countries that primarily speak another language $(\mathrm{P}=0.016)$ (Fig. 3$)$. This may imply that a language barrier plays a role in limiting the quality

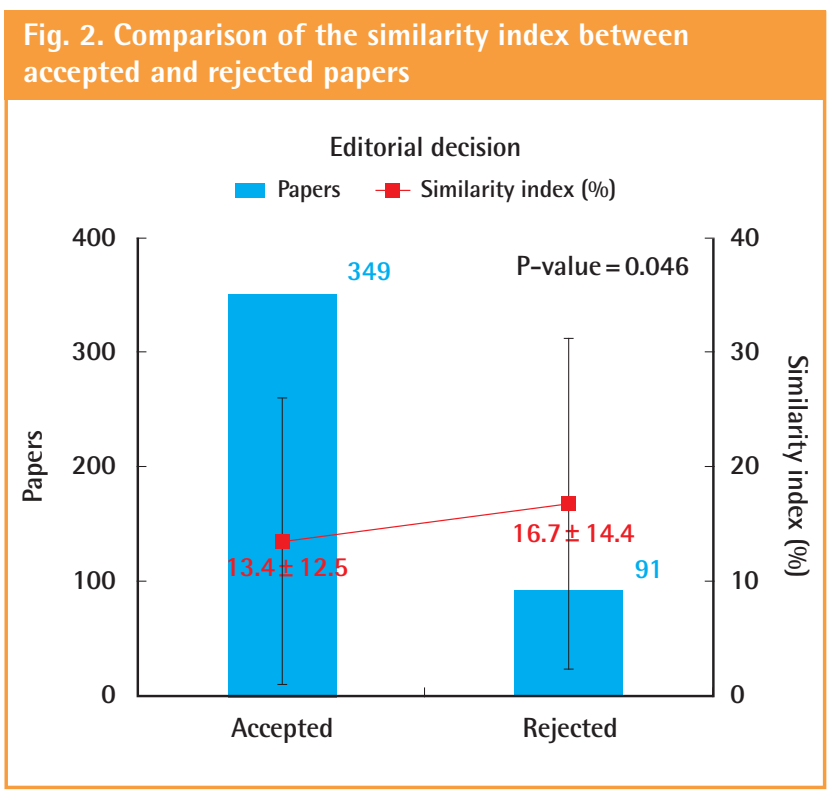

Copyright () 2014 The Korean Society of Plastic and Reconstructive Surgeons

This is an Open Access article distributed under the terms of the Creative Commons Attribution Non-Commercial License (http://creativecommons.org/

licenses/by-nc/3.0/) which permits unrestricted non-commercial use, distribution, and reproduction in any medium, provided the original work is properly cited. I www.e-aps.org 
Fig. 3. Comparison of the similarity index between Englishand non-English-language countries

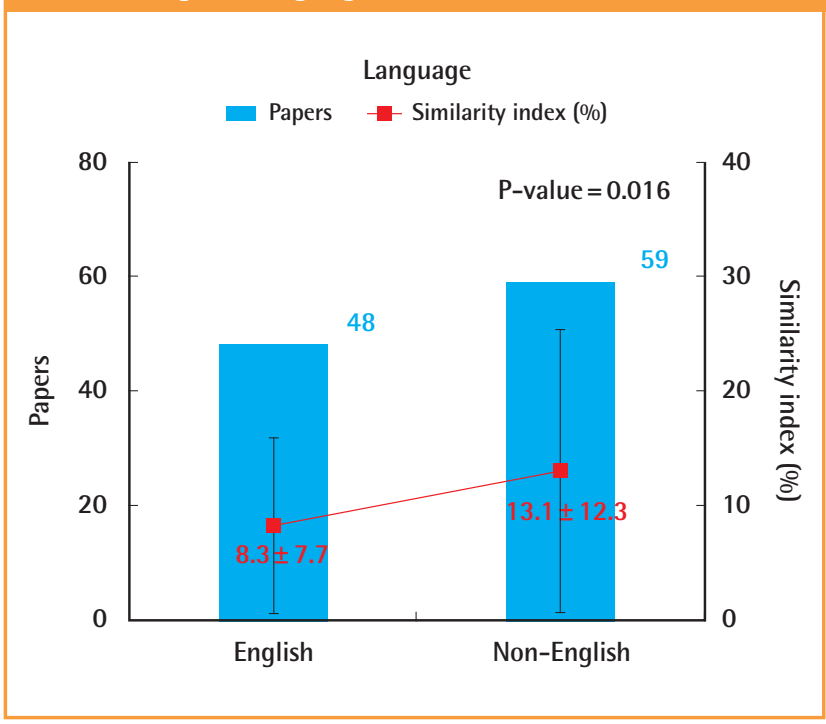

Fig. 5. Comparison of the similarity index between clinical and experimental papers

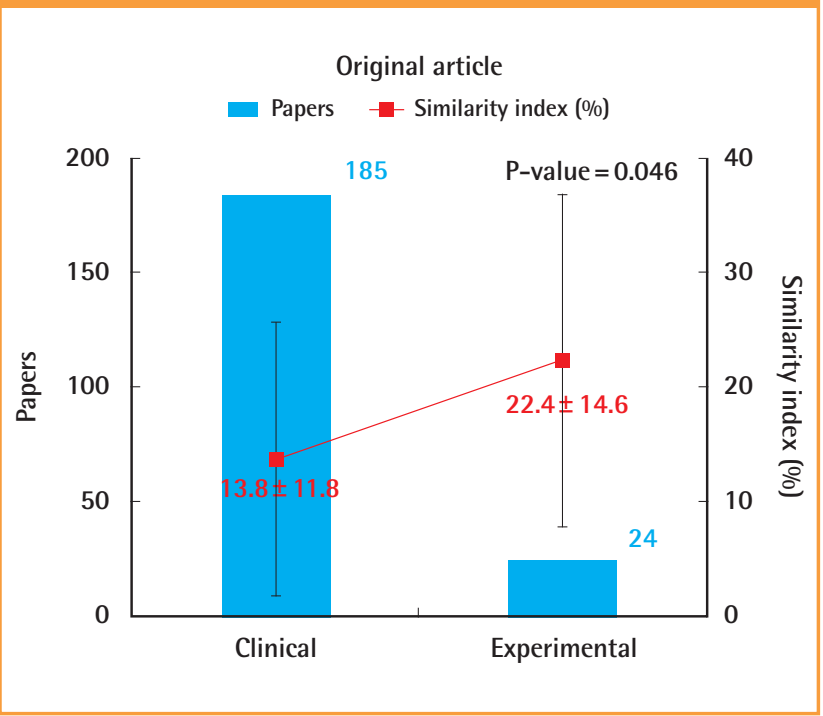

Publication type

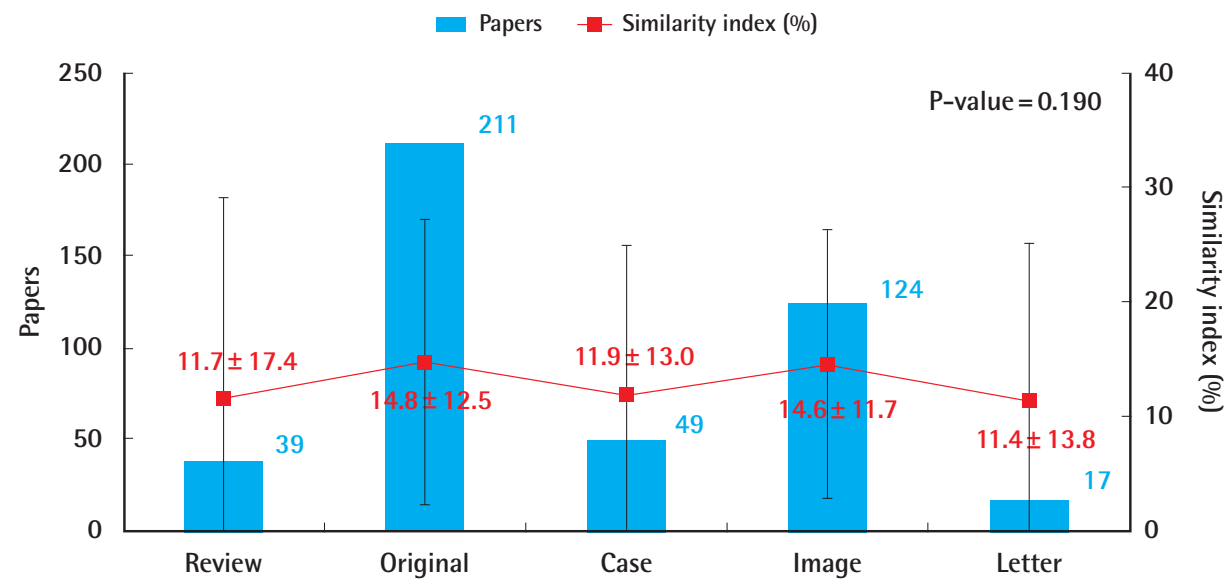

of papers. The similarity index of the original articles and image articles was higher than those of the other categories of articles, but the difference was not statistically significant $(\mathrm{P}=0.190)$ (Fig. 4). Among the original articles, the experimental papers had a higher similarity index than the clinical papers $(\mathrm{P}=0.001)$ (Fig. 5). Similar experimental methods among experimental papers may have led to such results, even for papers with legitimately original content.

Although we cannot jump to the conclusion that higher similarity index manuscripts are necessarily 'low-grade' manuscripts, higher similarity may imply that an article is a patchwork paper composed of sentences copied from other articles [2]. This is problematic even if the research methodology, data, and analysis are sound because the papers with a high similarity index may represent the lack of effort to write an original text. Since the statistical significance of the relationship between the acceptance of a manuscript and a low similarity index has been demonstrated, we must take action. This author recommends that papers submitted to the Archives of Plastic Surgery with a similarity index of over $40 \%$ be sent back to the authors to be rewritten. However, among the types of articles, experimental papers should be judged with greater flexibility due to their characteristic feature of high similarity. Authors who wish to submit a paper to this journal would be well served to use their own words and sentences in writing in order to ensure that they effectively communicate the quality and originality of their research. 


\section{REFERENCES}

1. Kim JT. The value of originality and proper quotation. Arch Plast Surg 2012;39:279-80.

2. Huh S. What happened when Crosscheck was not used for a month in Journal of Neurogastroenterology and Motility? J Neurogastroenterol Motil 2014;20:417-8.
Correspondence: Jang Hyun Lee

Department of Plastic and Reconstructive Surgery, Hanyang University Guri Hospital, Hanyang University College of Medicine, 153 Gyeongchun-ro, Guri 471-701, Korea

Tel: +82-31-560-2330, Fax: +82-31-560-2338, E-mail: pslee@ hanyang.ac.kr

No potential conflict of interest relevant to this article was reported.

Received: 29 Aug 2014 • Revised: 29 Aug 2014 • Accepted: 29 Aug 2014 pISSN: 2234-6163・ elSSN: 2234-6171

http://dx.doi.org/10.5999/aps.2014.41.5.449 • Arch Plast Surg 2014;41:449-451 